\title{
ASYMPTOTIC BEHAVIOR OF INCREMENTS OF RANDOM FIELDS
}

\author{
UDC 519.21
}

\author{
O. E. SHCHERBAKOVA
}

\begin{abstract}
Some results on the asymptotic behavior of increments of a $d$-dimensional random field are proved. Let $N$ and $a_{N} \in\{1,2, \ldots\}$ be fixed and let $S_{N}^{\star}$ be the maximum increment of a $d$-dimensional random field of independent identically distributed random variables evaluated for $d$-dimensional rectangles $(i, j]=\{k: i<k \leq j\}$ such that $|j| \leq N$ and $|j-i|=a_{N}$. Denote also by $S_{N}$ the maximum increment evaluated for rectangles such that $|j-i| \leq a_{N}$.

We determine the asymptotic almost sure behavior of random variables $S_{N}$ and $S_{N}^{\star}$. Steinebach (1983) proved a similar result for the case of rectangles belonging to the cube $\left(0, N^{1 / d}\right]$ (of volume $N$ ) and under the condition that $a_{N}=O\left(N^{\delta}\right)$ as $N \rightarrow \infty$ for all $\delta \in(0,1)$. Note that the sequence $S_{N}$ is monotone in this case.

We also consider the cases where $a_{N} \sim C \log N$ or $a_{N}=O(\log N)$.
\end{abstract}

\section{INTRODUCTION}

Let $\mathbf{N}_{0}^{d}$ be the $d$-dimensional $(d \geq 1)$ lattice whose elements have nonnegative integer coordinates. We introduce a partial order on $\mathbf{N}_{0}^{d}$ :

$$
i \leq j \quad \text { for } \quad i=\left(i_{1}, \ldots, i_{d}\right) \text { and } j=\left(j_{1}, \ldots, j_{d}\right)
$$

if and only if

$$
i_{k} \leq j_{k} \quad \text { for all } k=1, \ldots, d \text {. }
$$

Let

$$
(i, j]=\left\{k \in \mathbf{N}_{0}^{d}, i<k \leq j\right\}, \quad i, j \in \mathbf{N}_{0}^{d}, i \leq j,
$$

be a $d$-dimensional rectangle. The volume of the $d$-dimensional rectangle $(0, n]$ such that $n=\left(n_{1}, \ldots, n_{d}\right)$ and $n_{k} \in \mathbf{N} \cup\{0\}$ is denoted by $|n|=\prod_{i=1}^{d} n_{i}$.

Consider a sequence $X_{n}, n \in \mathbf{N}_{0}^{d}$, of independent identically distributed random variables depending on $d$ indices and with moment generating function $\phi(t)=\mathrm{E} \exp \left(t X_{n}\right)$ such that

$$
\phi(t)<\infty \text { for some } t>0 \text {. }
$$

We further assume that

$$
\mathrm{E} X_{n}=0, \quad \operatorname{Var} X_{n}=1 .
$$

Consider a function $a(t), t \geq 1, t \in \mathbf{R}$, such that

$$
1 \leq a(t) \leq t, \quad a(t) \text { and } \frac{t}{a(t)} \text { do not decrease. }
$$

Put $a_{N}=[a(N)]$, where $[\cdot]$ stands for the integer part of a real number.

2000 Mathematics Subject Classification. Primary 60F15; Secondary 60K05.

Supported in part by the Ministry of Education of the Russian Federation under grants N E00-1.0-82 and "Leading scientific school" \# 00-15-96019. 
Let $S_{N}^{\star}$ be the maximum increment of a $d$-dimensional field of independent identically distributed random variables evaluated for $d$-dimensional rectangles

$$
\{k: i<k \leq j\}
$$

such that $|j| \leq N$ and $|j-i|=a_{N}$. If the rectangles satisfy the inequality $|j-i| \leq a_{N}$ instead of the equality $|j-i|=a_{N}$, then the maximum increment is denoted by $S_{N}$ :

$$
\begin{gathered}
S_{(i, j]}=\sum_{i<k \leq j} X_{k}, \quad(i, j] \subset \mathbf{N}_{0}^{d}, \\
S_{N}^{\star}=\max _{\substack{|j| \leq N,|j-i|=a_{N}}} S_{(i, j]}, \quad S_{N}=\max _{\substack{|j| \leq N,|j-i| \leq a_{N}}} S_{(i, j]} .
\end{gathered}
$$

\section{SOME HISTORICAL REMARKS}

Csörgő and Révész [3] obtained limit theorems for increments of partial sums of independent identically distributed random variables in the case of $d=1$ by using corresponding results for the Wiener process and the strong invariance principle. They also considered the asymptotic behavior of increments of the two parameter Wiener process.

Below we recall some results for random variables with multiindices.

Theorem 2.1 (Steinebach [1). Let $X_{n}, n \in \mathbf{N}_{0}^{d}$, be independent identically distributed random variables. Assume that conditions (1) and (2) hold. If

$$
\begin{gathered}
\quad \frac{a_{N}}{\log N} \rightarrow \infty, \quad N \rightarrow \infty, \\
\frac{a_{N}}{N^{\delta}} \rightarrow 0, \quad N \rightarrow \infty, \quad \text { for all } \delta>0, \\
a_{N} \quad \text { does not decrease, } \\
\frac{a_{N}}{N^{\delta_{0}}} \quad \text { does not increase for some } \delta_{0},
\end{gathered}
$$

then

$$
\lim _{N \rightarrow \infty} \frac{D\left(N, a_{N}\right)}{\left(2 a_{N} \log N\right)^{1 / 2}}=\lim _{N \rightarrow \infty} \frac{D^{\star}\left(N, a_{N}\right)}{\left(2 a_{N} \log N\right)^{1 / 2}}=1 \quad \text { a.s. }
$$

where $D\left(N, a_{N}\right)$ is the maximum of increments evaluated on d-dimensional rectangles $J$, $|J| \leq a_{N}$, belonging to the d-dimensional cube $\left(0,\left[N^{1 / d}\right] \cdot e\right]$, and $e=(1, \ldots, 1)$ :

$$
D\left(N, a_{N}\right)=\max _{\substack{J \subset\left(0,\left[N^{1 / d}\right] \cdot e\right],|J| \leq a_{N}}} S_{J}, \quad D^{\star}\left(N, a_{N}\right)=\max _{\substack{J \subset\left(0,\left[N^{1 / d}\right] \cdot e\right] \\|J|=a_{N}}} S_{J} .
$$

Note that the volume of the cube $\left(0,\left[N^{1 / d}\right] \cdot e\right]$ is less than or equal to $N-d N^{1-1 / d}$.

Theorem 2.2 (Steinebach [1]). Assume that conditions (5)-(7) hold, and moreover

$$
a_{N} \sim C \log N, \quad N \rightarrow \infty,
$$

where $C$ is a unique solution of the equation

$$
\inf _{t \in\left[0, t_{0}\right)} \frac{\phi(t)}{\exp (\alpha t)}=\exp (-1 / C)
$$

for

and $t_{0}=\sup \{t: \phi(t)<\infty\}$. Then

$$
\alpha \in\left\{\frac{\phi^{\prime}(t)}{\phi(t)}: t \in\left[0, t_{0}\right)\right\}
$$

$$
\lim _{N \rightarrow \infty} \frac{D\left(N, a_{N}\right)}{\left(C \cdot a_{N} \log N\right)^{1 / 2}}=\lim _{N \rightarrow \infty} \frac{D^{\star}\left(N, a_{N}\right)}{\left(C \cdot a_{N} \log N\right)^{1 / 2}}=\alpha \quad \text { a.s. }
$$


Theorem 2.3 (Steinebach [1]). Assume that $\phi(t)<\infty$ for all $t>0$,

$$
\lim _{\tau \rightarrow+\infty} \log \phi^{\prime \prime}(\tau)=\gamma^{2}>0,
$$

and the sequence $a_{N}$ satisfies conditions (5) -(7) and

$$
\frac{a_{N}}{\log N} \rightarrow 0, \quad N \rightarrow \infty
$$

Then

$$
\lim _{N \rightarrow \infty} \frac{D\left(N, a_{N}\right)}{\left(2 \gamma^{2} a_{N} \log N\right)^{1 / 2}}=\lim _{N \rightarrow \infty} \frac{D^{\star}\left(N, a_{N}\right)}{\left(2 \gamma^{2} a_{N} \log N\right)^{1 / 2}}=1 \quad \text { a.s. }
$$

\section{Main Results}

The main goal of this paper is to study the asymptotic behavior of the maximum of increments of a $d$-dimensional random field evaluated for rectangles whose volumes do not exceed $a_{N}$ under the condition that all of them belong to a rectangle of volume $N$. In other words, we study the asymptotic behavior of random variables $S_{N}$ and $S_{N}^{\star}$ introduced in Section 1.

Theorem 3.1. Let conditions (11)-(31) hold. Assume that

$$
\frac{a_{N}}{\log N} \rightarrow \infty, \quad N \rightarrow \infty .
$$

Put $\delta_{N}=\left\{2 a_{N}\left(\varepsilon_{N}+\beta_{N}\right)\right\}^{-1 / 2}$, where

$$
\varepsilon_{N}=\log _{+} \frac{N}{a_{N}}, \quad \beta_{N}=d \log \log N,
$$

and $\log _{+} x=\log (x \vee e)$. Then

$$
\limsup _{N \rightarrow \infty} S_{N}^{\star} \delta_{N}=\limsup _{N \rightarrow \infty} S_{N} \delta_{N}=1 \quad \text { a.s. }
$$

If additionally

$$
\frac{\varepsilon_{N}}{\beta_{N}} \rightarrow \infty, \quad N \rightarrow \infty
$$

then

$$
\lim _{N \rightarrow \infty} S_{N}^{\star} \delta_{N}=\lim _{N \rightarrow \infty} S_{N} \delta_{N}=\lim _{N \rightarrow \infty} D\left(N, a_{N}\right) \delta_{N}=\lim _{N \rightarrow \infty} D^{\star}\left(N, a_{N}\right) \delta_{N}=1 \quad \text { a.s. }
$$

Remark 3.1. Condition (13) holds for all sequences $\left\{a_{N}\right\}$ such that

$$
a_{N}=O\left(N^{\delta_{0}}\right), \quad N \rightarrow \infty, \quad \text { for some } \delta_{0} \in(0,1) .
$$

Theorem 3.2. Assume that

$$
a_{N} \sim C \log N, \quad N \rightarrow \infty,
$$

where the constant $C$ is defined in Theorem 2.2 (see (9)). Let

$$
\delta_{N}=\left\{2 C a_{N} \log N\right\}^{-1 / 2} .
$$

Then

$$
\lim _{N \rightarrow \infty} S_{N} \delta_{N}=\lim _{N \rightarrow \infty} S_{N}^{\star} \delta_{N}=\lim _{N \rightarrow \infty} D^{\star}\left(N, a_{N}\right) \delta_{N}=\lim _{N \rightarrow \infty} D\left(N, a_{N}\right) \delta_{N}=\alpha \quad \text { a.s. }
$$


Theorem 3.3. Assume that $\phi(t)<\infty$ for all $t>0$,

$$
\lim _{\tau \rightarrow+\infty} \log \phi^{\prime \prime}(\tau)=\gamma^{2}>0
$$

and

$$
\frac{a_{N}}{\log N} \rightarrow 0, \quad N \rightarrow \infty
$$

Put

Then

$$
\delta_{N}=\left\{2 \gamma^{2} a_{N} \log N\right\}^{-1 / 2} .
$$

$$
\lim _{N \rightarrow \infty} S_{N} \delta_{N}=\lim _{N \rightarrow \infty} S_{N}^{\star} \delta_{N}=\lim _{N \rightarrow \infty} D^{\star}\left(N, a_{N}\right) \delta_{N}=\lim _{N \rightarrow \infty} D\left(N, a_{N}\right) \delta_{N}=1 \quad \text { a.s. }
$$

\section{Auxiliary Results}

We need some combinatorial estimates. In what follows let $d \geq 2$. We denote by $P_{d-1}$ polynomials of degree $d-1$.

Lemma 4.1. Put

$$
B_{d}(n)=\operatorname{Card}\left\{(0, n] \subset \mathbf{N}_{0}^{d}:|n|=N\right\} .
$$

Then for all $\varepsilon>0$

$$
\sum_{n \leq N} B_{d}(n)=N \cdot P_{d-1}(\log N)+O\left(N^{(d-1) /(d+2)+\varepsilon}\right) .
$$

Remark 4.1. It is clear that $B_{d}(N)$ equals the total number of representations of the number $N$ as a product of $d$ integer factors. The proof of (18) can be found in [4] (also see [6]).

We also need an estimate for some coverings $A_{N, a_{N}}^{\square}$ of the set

$$
A(N)=\bigcup_{|n| \leq N}(0, n]
$$

by $d$-dimensional rectangles whose volumes are equal to $a_{N}$.

Lemma 4.2. Let $N \geq 1$ and a vector $i^{0} \in \mathbf{N}_{0}^{d}$ be such that $\left|i^{0}\right|=a_{N}$. Further let $A_{N, a_{N}}^{\square}$ be the minimal covering of $A(N)$ by disjoint rectangles obtained by parallel translations of the vector $i^{0}$. In other words,

$$
A_{N, a_{N}}^{\square}=\left\{\left(j(l)-i^{0}, j(l)\right] \subset A(N): l \in \mathbf{N}_{0}^{d}, j_{k}\left(l_{k}\right)=l_{k} i_{k}^{0}, l_{k} \in\left\{1, \ldots,\left[\frac{n_{k}}{i_{k}^{0}}\right]+1\right\}\right\},
$$

where $n_{k}$ is the maximum of the $k$ th coordinates of vectors $n$ such that $i^{0} \leq n$ and $|n|=N, k=1, \ldots, d$. Then

$$
\begin{gathered}
\operatorname{Card}\left(A_{N, 1}^{\square}\right)=\sum_{n \leq N} B_{d}(n) \leq N \cdot P_{d-1}(\log N), \\
\quad \operatorname{Card}\left(A_{N, a_{N}}^{\square}\right) \leq \frac{N}{a_{N}} \cdot P_{d-1}\left(\log _{+} \frac{N}{a_{N}}\right) .
\end{gathered}
$$

Proof of Lemma 4.2 First we consider the case of $a_{N}=1$. Applying Lemma 4.1 we obtain

$$
\operatorname{Card}\left(A_{N, 1}^{\square}\right)=\sum_{n \leq N} B_{d}(n)=N \cdot P_{d-1}(\log N)+O\left(N^{(d-1) /(d+2)+\varepsilon}\right) .
$$

In the case of $a_{N}>1$ we consider the mapping $x_{k}=x_{k} / i_{k}^{0}, k=1, \ldots, d$. If a rectangle of $A_{N, a_{N}}^{\square}$ is of volume $a_{N}$, then this mapping transforms it into a cube of unit volume. Note 
also that the set $A(N)$ is transformed into the set $A\left(N / a_{N}\right)$ under this mapping, and thus Card $A_{N, a_{N}}^{\square}=\operatorname{Card} A_{N / a_{N}, 1}^{\square}$ and $\operatorname{Card}\left(A_{N, a_{N}}^{\square}\right) \leq\left(N / a_{N}\right) P_{d-1}\left(\log _{+}\left(N / a_{N}\right)\right)$.

The following result allows one to estimate the cardinality of the collection of rectangles of $A(N)$ whose volumes do not exceed $a_{N}$.

Lemma 4.3. Let

$$
A_{N}=\left\{(i, j] \subset A(N):|i-j| \leq a_{N}\right\}
$$

Then

$$
\operatorname{Card}\left(A_{N}\right) \leq N \cdot a_{N} \cdot P_{d-1}\left(\log _{+} a_{N}\right) \cdot P_{d-1}(\log N)
$$

for all sufficiently large $a_{N}$.

Proof of Lemma 4.3. The total number of rectangles of $(0, l]$ whose volumes do not exceed $N$ is equal to $\operatorname{Card}\left(A_{N, 1}^{\square}\right)$. For any given $l$, there are at most $\operatorname{Card}\left(A_{a_{N}, 1}^{\square}\right)$ rectangles $(l, k]$ such that $|k-l|=a_{N}$. Hence

$\operatorname{Card}\left(A_{N}\right)=\operatorname{Card}\left(A_{N, 1}^{\square}\right) \cdot \operatorname{Card}\left(A_{a_{N, 1}}^{\square}\right) \leq N \cdot a_{N} \cdot P_{d-1}\left(\log _{+} a_{N}\right) \cdot P_{d-1}(\log N)$.

Denote by $\mathbf{R}_{0}^{d}$ the space of $d$-dimensional vectors with nonnegative real coordinates.

Lemma 4.4. For all $\varrho>1$ and all natural numbers $a_{N}>\varrho$ there is a finite set

$$
U_{\varrho}\left(a_{N}\right) \subset \mathbf{R}_{0}^{d}
$$

such that

(1) $|u|=\varrho^{d-1} \cdot a_{N}$ for all $u \in U_{\varrho}\left(a_{N}\right)$;

(2) for all $i \in \mathbf{N}_{0}^{d},|i| \leq a_{N}$, there exists $u \in U_{\varrho}\left(a_{N}\right)$ such that $i \leq u$;

(3) $\operatorname{Card}\left(U_{\varrho}\left(a_{N}\right)\right)=\left[\left(\log ^{d-1} a_{N}\right) /\left(\log ^{d-1} \varrho\right)\right]$.

Proof. Let $\varrho>1$. For $i \in \mathbf{N}_{0}^{d}$ such that $|i| \leq a_{N}$ we put

$$
\begin{aligned}
U_{\varrho}\left(a_{N}\right)=\left\{u_{k}=\right. & \left(u_{k_{1}}, \ldots, \frac{a_{N}}{\prod_{s=1}^{d-1} u_{k_{s}}}\right)=\left(\varrho^{k_{1}+1}, \ldots, \varrho^{k_{d-1}+1}, \frac{a_{N}}{\varrho^{\sum_{s=1}^{d-1} k_{s}}}\right), \\
& \left.k_{s} \in\left\{1, \ldots,\left[\frac{\log a_{N}}{\log \varrho}\right]\right\}, s=1, \ldots, d-1,\left|u_{k}\right|=\varrho^{d-1} \cdot a_{N}\right\} .
\end{aligned}
$$

There exists $k=\left(k_{1}, \ldots, k_{d-1}\right) \in \mathbf{N}_{0}^{d-1}$ such that $i \leq u_{k}$. Indeed, let $k_{s}=\left[\log i_{s} / \log \varrho\right]$. Then $\varrho^{k_{s}} \leq i_{s}<\varrho^{k_{s}+1}, s=1, \ldots, d-1$,

$$
i_{d} \leq \frac{a_{N}}{\sum_{s=1}^{d-1} i_{s}} \leq \frac{a_{N}}{\varrho^{\sum_{s=1}^{d-1} k_{s}}} .
$$

It is clear that all the assumptions of Lemma 4.3 are satisfied.

Lemma 4.5. For all $\varrho>1,0<\nu<1-1 / \varrho$, and for all natural numbers $N>\rho$ one can construct a finite set $V_{\varrho, \nu}\left(N, a_{N}\right) \subset \mathbf{R}_{0}^{d}$ such that

(1) for all $v \in V_{\varrho, \nu}\left(N, a_{N}\right)$, the volume of $v$ is equal to $\varrho^{2 d-1} \cdot a_{N}$;

(2) for all $(i-j, i] \subset \mathbf{N}_{0}^{d},|j| \leq a_{N}$ and $|i| \leq N$, there exists $v \in V_{d}\left(N, a_{N}\right)$ such that $(i-j, i] \subset v$;

(3) we have

$$
\operatorname{Card}\left(V_{\varrho, \nu}\left(N, a_{N}\right)\right) \leq \frac{\log _{N}^{d-1}}{\log ^{d-1} \varrho} \cdot \frac{N}{\nu^{d} \varrho^{d} a_{N}} \cdot P_{d-1}\left(\log _{+} \frac{N}{\nu^{d} \varrho^{d} a_{N}}\right)
$$


Proof. The set $V_{\varrho, \nu}\left(N, a_{N}\right)$ is constructed in the following way:

$$
\begin{gathered}
V_{\varrho, \nu}\left(N, a_{N}\right)=\left\{v=\left(w(l)-\varrho^{d} \cdot u, w(l)\right], u \in U_{\varrho}\left(a_{N}\right), l \in \mathbf{N}_{0}^{d},\right. \\
\left.w_{k}\left(l_{k}\right)=\nu \varrho l_{k} u_{k}, l_{k} \in\left\{1, \ldots,\left[\frac{n_{k}}{\nu \varrho u_{k}}\right]+1\right\}\right\},
\end{gathered}
$$

where $n_{k}$ is the maximum of the $k$ th coordinates of vectors $n \in U_{\varrho}(N)$ such that $u \leq n$, $k=1, \ldots, d$. The first assertion of the lemma is obvious.

Now we prove the second assertion. Let $(i-j, i] \subset \mathbf{N}_{0}^{d},|j| \leq a_{N}$ and $|i| \leq N$. According to Lemma 4.4. there are $u \in U_{\varrho}\left(a_{N}\right)$ and $n \in U_{\varrho}(N)$ such that $j<u$ and $u \leq n$, respectively.

Further, for any given $i$ there are $w(l): l=\left(l_{1}, \ldots, l_{d}\right) \in \mathbf{N}_{0}^{d}$ such that

$$
\nu \varrho\left(l_{k}-1\right) u_{k} \leq i_{k} \leq \nu \varrho l_{k} u_{k}=w_{k}\left(l_{k}\right) .
$$

Thus $\nu \varrho\left(l_{k}-1\right) u_{k}-u_{k} \leq i_{k}-u_{k} \leq \nu \varrho l_{k} u_{k}-u_{k}$. Now we check that

$$
i_{k}-u_{k} \geq\left(\nu l_{k}-1\right) \varrho u_{k} .
$$

This inequality holds if $\nu \varrho\left(l_{k}-1\right) u_{k}-u_{k} \geq\left(\nu l_{k}-1\right) \varrho u_{k}$. The latter condition is equivalent to $\nu \varrho \leq \varrho-1$, which is true by construction.

Now we prove the third assertion of the lemma. Note that the cardinality of the set $V_{\varrho, \nu}\left(N, a_{N}\right)$ depends on the indices $u$ and $l$. Since the sets of $d$-dimensional indices $l$ used in the definitions of $A_{N, a_{N}}^{\square}$ and $V_{\varrho, \nu}\left(N, a_{N}\right)$ coincide,

$$
\operatorname{Card}\left(V_{\varrho, \nu}\left(N, a_{N}\right)\right) \leq \frac{\log ^{d-1} a_{N}}{\log ^{d-1} \varrho} \cdot \frac{N}{\nu^{d} \varrho^{d} a_{N}} \cdot P_{d-1}\left(\log _{+} \frac{N}{\nu^{d} \varrho^{d} a_{N}}\right) .
$$

\section{Proofs of main Results}

Proof of Theorem [3.1. We split the proof into three steps.

Step 1. First we show that $\lim \sup S_{N} \delta_{N} \leq 1$ a.s. Consider an arbitrary $\varepsilon>0$. Using the above lemmas and applying Kolmogorov's inequality for random fields [5] we obtain

$$
\mathrm{P}\left(\max _{(0, i]:|i| \leq a_{N}} S_{(0, i]} \delta_{N}>1+2 \varepsilon\right) \leq 2^{d} \sum_{i^{0}: i^{0} \in U_{\varrho}\left(a_{N}\right)} \mathrm{P}\left(S_{\left(0, i^{0}\right]}>(1+\varepsilon) \delta_{N}^{-1}\right) .
$$

Further

$$
\begin{aligned}
\mathrm{P}\left(S_{N}>(1+2 \varepsilon) \delta_{N}^{-1}\right) \leq 2^{d} \cdot \sum_{(j-i, j] \in V_{\varrho, \nu}\left(N, a_{N}\right)} \mathrm{P}\left(S_{(j-i, j]}>(1+\varepsilon) \delta_{N}^{-1}\right) \\
=2^{d} \cdot \sum_{(j-i, j] \in V_{\varrho, \nu}\left(N, a_{N}\right)} \mathrm{P}\left(\sqrt{\frac{\varepsilon_{N}+\beta_{N}}{a_{N}}} S_{(j-i, j]}>(1+\varepsilon) \delta_{N}^{-1} \sqrt{\frac{\varepsilon_{N}+\beta_{N}}{a_{N}}}\right) .
\end{aligned}
$$

Note that

Moreover

$$
\delta_{N}^{-1} \cdot \sqrt{\frac{\varepsilon_{N}+\beta_{N}}{a_{N}}}=\sqrt{2}\left(\varepsilon_{N}+\beta_{N}\right) .
$$

$$
\frac{\varepsilon_{N}+\beta_{N}}{a_{N}}=\frac{\log N}{a_{N}}-\frac{\log a_{N}}{a_{N}}+\frac{d \log \log N}{a_{N}} \rightarrow 0, \quad N \rightarrow \infty .
$$

Expanding the function

$$
\log \phi\left(\sqrt{\frac{\varepsilon_{N}+\beta_{N}}{a_{N}}} \cdot t\right)
$$


in the Taylor series, we get

$$
\lim _{N \rightarrow \infty} \frac{\varrho^{2 d-1} \cdot a_{N}}{\varepsilon_{N}+\beta_{N}} \cdot \log \phi\left(\sqrt{\frac{\varepsilon_{N}+\beta_{N}}{a_{N}}} \cdot t\right)=\frac{\varrho^{2 d-1} \cdot t^{2}}{2} .
$$

We recall a theorem due to Plachky and Steinebach [2].

Theorem 5.1. Let $\left\{W_{n}\right\}_{n=1,2, \ldots}$ be a sequence of random variables such that

(1) $m_{n}(t)=\int \exp \left(t W_{n}\right) d \mathrm{P}<\infty$ for all $t \in\left(0, T_{1}\right), T_{1}>0$,

(2) $\psi_{n}(t) / n \rightarrow c_{0}(t)$ for all $t \in\left(T_{0}, T_{1}\right), 0 \leq T_{0}<T_{1}$, where $\psi_{n}(t)=\log m_{n}(t)$.

Then

$$
\lim \left(\mathrm{P}\left(W_{n}>n a_{n}\right)\right)^{1 / n}=\inf _{t>0}\left\{\exp \left(c_{0}(t)-a t\right)\right\}
$$

for every sequence $\left\{a_{n}\right\}_{n=1,2, \ldots}, a_{n} \in \mathbf{R}$, such that

$$
\begin{aligned}
& a_{n} \rightarrow a \in A=\left\{c_{0}^{\prime}(h): c_{0}^{\prime}(h)\right. \text { exists, is right continuous, } \\
& \text { and is strictly monotone for } \left.h \in\left(T_{0}, T_{1}\right)\right\} .
\end{aligned}
$$

In what follows we need a more general result under a weaker condition than (2).

Remark 5.1. In the case under consideration

$$
\begin{gathered}
n=\varepsilon_{N}+\beta_{N}, \quad W_{n}=\sqrt{\frac{\varepsilon_{N}+\beta_{N}}{a_{N}}} S_{(j-i, j]}, \\
m_{n}(t)=\mathrm{E}\left(\exp \left(t \sqrt{\frac{\varepsilon_{N}+\beta_{N}}{a_{N}}} S_{(j-i, j]}\right)\right), \quad a_{n}=\sqrt{2}(1+\varepsilon), \\
c_{2}(t)=\frac{t^{2}}{2}=\liminf \psi_{n}(t) / n \leq \lim \sup \psi_{n}(t) / n=\frac{\varrho^{2 d-1} \cdot t^{2}}{2}=c_{1}(t) .
\end{gathered}
$$

Therefore condition (2) of Theorem 5.1 does not hold and the function $c_{0}(t)$ is not well defined. Nevertheless

$$
\begin{aligned}
A_{2} & =\inf _{t>0}\left(\exp \left\{\frac{t^{2}}{2}-\sqrt{2} t(1+\varepsilon)\right\}\right)=\exp \left\{-(1+\varepsilon)^{2}\right\} \\
& =\liminf \mathrm{P}\left(\sqrt{\frac{\varepsilon_{N}+\beta_{N}}{a_{N}}} S_{(j-i, j]}>(1+\varepsilon) \sqrt{2}\left(\varepsilon_{N}+\beta_{N}\right)\right)^{\frac{1}{\varepsilon_{N}+\beta_{N}}} \\
& \leq \limsup \mathrm{P}\left(\sqrt{\frac{\varepsilon_{N}+\beta_{N}}{a_{N}}} S_{(j-i, j]}>(1+\varepsilon) \sqrt{2}\left(\varepsilon_{N}+\beta_{N}\right)\right)^{\frac{1}{\varepsilon_{N}+\beta_{N}}} \\
& =\inf _{t>0}\left(\exp \left\{\frac{\varrho^{2 d-1} \cdot t^{2}}{2}-\sqrt{2} t(1+\varepsilon)\right\}\right)=\exp \left\{-(1+\varepsilon)^{2} / \varrho^{2 d-1}\right\}=A_{1} .
\end{aligned}
$$

Proof. Assume the opposite. Put $P_{n}=\left(\mathrm{P}\left(W_{n}>n a_{n}\right)\right)^{1 / n}$. Note that the sequence $\psi_{n}^{\prime}(t) / n$ is uniformly bounded for $t \in[0, T]$ and all sufficiently small $T>0$. Indeed,

Moreover

$$
\psi_{n}^{\prime}(t) / n \leq \varrho^{2 d-1} t \frac{\mathrm{E} X e^{h X}}{h \mathrm{E} e^{h X}}, \quad h=t \sqrt{\frac{\varepsilon_{N}+\beta_{N}}{a_{N}}} \rightarrow 0 .
$$

$$
\begin{aligned}
& E\left(X e^{h X}\right)=\int_{-\infty}^{0} x\left(e^{h x}-1\right) d F(x)+\int_{0}^{\infty} x\left(e^{h x}-1\right) d F(x) \\
&<h\left(\int_{-\infty}^{0} x^{2} d F(x)+\int_{0}^{\infty} x^{2} e^{h x} d F(x)\right), \\
& e^{y}-1<y e^{y}, \quad e^{-y}-1>-y, \quad y>0
\end{aligned}
$$


by $\mathrm{E} X=0$ and $\operatorname{Var} X=1$. Therefore $\mathrm{E}\left(X e^{h X}\right) \leq c \cdot h, t \in[0, T]$, implying that $\psi_{n}^{\prime}(t) / n$ is uniformly bounded.

Now we assume that there exists a subsequence $P_{n_{k}}$ such that

$$
\lim P_{n_{k}} \notin\left[A_{2}, A_{1}\right] .
$$

Consider an infinite collection of increasing uniformly bounded functions $\Psi=\left\{\psi_{n_{k}}^{\prime} / n_{k}\right\}$, $\psi_{n_{k}}^{\prime} / n_{k}:[0, T] \mapsto \mathbf{R}$. By Lemma 2, 4.VIII in [7] there exists a subcollection

$$
\left\{\psi_{n_{k_{m}}}^{\prime} / n_{k_{m}}\right\} \subset \Psi
$$

such that the limit

$$
\lim _{m \rightarrow \infty} \psi_{n_{k_{m}}}^{\prime} / n_{k_{m}}(t)=c_{\star}(t)
$$

exists for all $t \in[0, T]$ and the function $c_{\star}(t)$ is nondecreasing and right continuous. Moreover the function $c_{\star}(t)$ is strictly increasing, since the functions $\psi_{n}^{\prime \prime}(t) / n$ are positive and bounded away from zero and infinity for all sufficiently large $n$ and $t \in[0, T]$. Put

$$
c_{0}(t)=\lim _{n_{k_{m}} \rightarrow \infty} \int_{0}^{t} \psi_{n_{k_{m}}}^{\prime}(x) / n_{k_{m}} d x .
$$

By the Lebesgue theorem (see Theorem 16.3 in 8 ) we get $c_{0}(t)=\int_{0}^{t} c_{\star}(x) d x$, whence $c_{0}^{\prime}(t)=c_{\star}(t)$.

Thus by the Plachky and Steinebach theorem

$$
\lim \left(\mathrm{P}\left(W_{n_{k_{p}}}>n_{k_{p}} a\right)\right)^{1 / n_{k_{p}}}=\inf _{t>0}\left\{\exp \left(c_{0}(t)-a t\right)\right\} \in\left[A_{2}, A_{1}\right]
$$

and we arrive at a contradiction.

Using Lemma 4.5, Remark [5.1, and relation (19) we get

$$
\begin{aligned}
\mathrm{P}\left(S_{N} \delta_{N}>1+2 \varepsilon\right) \leq & 2^{d-1} \operatorname{Card}\left(V_{\varrho}\left(N, a_{N}\right)\right) \exp \left(-\frac{(1+\varepsilon / 2)^{2}}{\varrho^{2 d-1}}\left(\varepsilon_{N}+\beta_{N}\right)\right) \\
\leq & \frac{\log ^{d-1} a_{N}}{\nu^{d} \varrho^{d} \log ^{d-1} \varrho} \cdot\left(\frac{N}{a_{N}}\right)^{1-(1+\varepsilon / 2)^{2} / \varrho^{2 d-1}} \\
& \times P_{d-1}\left(\log _{+} \frac{N}{\nu^{d} \varrho^{d} a_{N}}\right) \cdot \log ^{-d(1+\varepsilon / 2)^{2} / \varrho^{2 d-1}} N .
\end{aligned}
$$

Assume that $(1+\varepsilon / 2)^{2}>\varrho^{2 d-1}$. Note that the sequence

$$
\left(\frac{N}{a_{N}}\right)^{1-(1+\varepsilon / 2)^{2} / \varrho^{2 d-1}} \cdot P_{d-1}\left(\log _{+} \frac{N}{\nu^{d} \varrho^{d} a_{N}}\right)
$$

is bounded.

Let $\vartheta>1$ and $N=\left[\vartheta^{r}\right]$. The series

$$
\sum_{r=1}^{\infty} \mathrm{P}\left(S_{\left[\vartheta^{r}\right]} \delta_{\left[\vartheta^{r}\right]}>1+2 \varepsilon\right)
$$

converges, since its general term is of order

$$
r^{-1-d\left((1+\varepsilon / 2)^{2} /\left(\varrho^{2 d-1}\right)-1\right)} .
$$

Letting $\varepsilon \rightarrow 0$ and $\varrho \rightarrow 1$ and applying the Borel-Cantelli lemma we obtain

$$
\limsup S_{\left[\vartheta^{r}\right]} \delta_{\left[\vartheta^{r}\right]} \leq 1 \quad \text { a.s. for all } \vartheta>1 .
$$


For any $N \in \mathbf{N}$, there exists $r$ such that $\left[\vartheta^{r}\right] \leq N<\left[\vartheta^{r+1}\right]$ and

$$
S_{N}^{\star} \delta_{N} \leq S_{N} \delta_{N} \leq S_{\left[\vartheta^{r+1}\right]} \delta_{\left[\vartheta^{r+1}\right]} \frac{\delta_{\left[\vartheta^{r}\right]}}{\delta_{\left[\vartheta^{r+1}\right]}}, \quad \limsup \frac{\delta_{\left[\vartheta^{r}\right]}}{\delta_{\left[\vartheta^{r+1}\right]}}=\sqrt{\vartheta}, \quad r \rightarrow \infty
$$

Thus

$$
\lim \sup S_{N}^{\star} \delta_{N} \leq \lim \sup S_{N} \delta_{N} \leq 1 \quad \text { a.s. }
$$

by letting $\vartheta \rightarrow 1$. This completes the proof of Step 1 .

Step 2. We show that

$$
\limsup _{N \rightarrow \infty} S_{N} \geq \limsup _{N \rightarrow \infty} S_{N}^{\star} \geq 1 \quad \text { a.s. }
$$

Choose $\varsigma$ such that $1>\varsigma>0$ and prove that

$$
\limsup _{N \rightarrow \infty} S_{N}^{\star} \geq 1-\varsigma \quad \text { a.s. }
$$

It is evident that the limit

$$
\lim _{t \rightarrow \infty} a(t) / t=p
$$

exists. First we consider the case of $p=0$. We construct the sequence $\left\{N_{k}\right\}_{k \in \mathbf{N}}$ as follows: for $\varsigma / 8>\varepsilon>0$ and

$$
0<\lambda<2^{1-d} \frac{\varsigma / 4}{1-\varsigma / 4}
$$

we choose $N_{1}$ in such a way that $a_{N} / N<\lambda$ for all $N>N_{1}$, and put

$$
\left[N_{k} / \varepsilon\right]=N_{k+1}-a_{N_{k+1}} .
$$

Now we construct a set of disjoint $d$-dimensional rectangles belonging to the domain $A\left(N_{k}\right) \backslash A\left(N_{k-1}\right)$ :

$$
\begin{array}{r}
\Im_{N_{k}}=\left\{J=(j, i] \in \mathbf{R}_{0}^{d}: i=\left(\varrho^{s_{1}}, \ldots, \varrho^{s_{d-1}} \frac{N_{k}}{\varrho^{\sum_{l=1}^{d-1} s_{l}}}\right), s_{l} \in\left\{1, \ldots,\left[\frac{\log N_{k}}{\log \varrho}\right]\right\}\right. \\
\left.s \in \mathbf{N}_{0}^{d-1}, l=1, \ldots, d-1, j=\left(\varrho^{s_{1}-1}, \ldots, \varrho^{s_{d-1}-1}, \frac{N_{k-1}}{\varrho^{\sum_{l=1}^{d-1} s_{l}-d+1}}\right)\right\}
\end{array}
$$

where

$$
\varrho=1+\left(a_{N_{k}} /\left(N_{k}-a_{N_{k}}\right)\right)^{1 /(d-1)} .
$$

Note that $|j|=N_{k-1}$ and $|i|=N_{k}$. Our current goal is to estimate $|i-j|$ for $(j, i] \in \Im_{N_{k}}$. 
We have

$$
\begin{aligned}
& |i-j|=(\varrho-1)^{d-1}\left(\frac{N_{k}}{\varrho^{d-1}}-N_{k-1}\right) \\
& =\frac{a_{N_{k}}}{N_{k}-a_{N_{k}}}\left(\frac{N_{k}}{\left(1+\left(a_{N_{k}} /\left(N_{k}-a_{N_{k}}\right)\right)^{1 /(d-1)}\right)^{d-1}}-N_{k-1}\right) \\
& =\frac{a_{N_{k}}}{N_{k}-a_{N_{k}}}\left(\frac{N_{k}-a_{N_{k}}}{\left(\left(1-a_{N_{k}} / N_{k}\right)^{1 /(d-1)}+\left(a_{N_{k}} / N_{k}\right)^{1 /(d-1)}\right)^{d-1}}-N_{k-1}\right) \\
& \geq a_{N_{k}}\left(\frac{1}{\left(1+\sum_{l=1}^{d-2} C_{d-1}^{l}\left(a_{N_{k}} / N_{k}\right)^{l /(d-1)}\left(1-a_{N_{k}} / N_{k}\right)^{(d-1-l) /(d-1)}\right)}-2 \varepsilon\right) \\
& >a_{N_{k}}\left(\frac{1}{1+\lambda 2^{d-1}}-2 \varepsilon\right)>a_{N_{k}}\left(\frac{1}{1+(\varsigma / 4) /(1-\varsigma / 4)}-2 \varepsilon\right)>a_{N_{k}}(1-\varsigma / 2) .
\end{aligned}
$$

Using the inequality $\log (1+x)<x$, we prove that

$$
\operatorname{Card}\left(\Im_{N_{k, 1}}\right) \geq \frac{\log ^{d-1} N_{k}}{2 \log ^{d-1}\left(1+\left(a_{N_{k}} /\left(N_{k}-a_{N_{k}}\right)\right)^{1 /(d-1)}\right)^{d-1}} \geq \frac{N_{k}-a_{N_{k}}}{2 a_{N_{k}}} \log ^{d-1} N_{k} .
$$

By Remark 5.1 we obtain $n=\varepsilon_{N}+\beta_{N},(j, i] \in \Im_{N_{k}}$,

$$
\begin{gathered}
W_{n}=\sqrt{\frac{\varepsilon_{N}+\beta_{N}}{a_{N}}} S_{(j, i]}, \quad m_{n}(t)=\mathrm{E}\left(\exp \left(t \sqrt{\frac{\varepsilon_{N}+\beta_{N}}{a_{N}}} S_{(j, i]}\right)\right), \\
\lim _{N \rightarrow \infty} \frac{(1-\varsigma / 2) \cdot a_{N}}{\varepsilon_{N}+\beta_{N}} \cdot \log \phi\left(\sqrt{\frac{\varepsilon_{N}+\beta_{N}}{a_{N}}} \cdot t\right)=\frac{(1-\varsigma / 2) \cdot t^{2}}{2}, \\
c_{2}(t)=\frac{(1-\varsigma / 2) t^{2}}{2}=\liminf \psi_{n}(t) / n .
\end{gathered}
$$

Therefore

$$
\begin{aligned}
\mathrm{P}\left(\max _{J \in \Im_{N_{k}}} S_{J} \delta_{N_{k}} \geq 1-\varsigma\right) & \geq 1-\left(1-\mathrm{P}\left(\mathrm{S}_{J J \in \Im_{N_{k}}} \delta_{N_{k}} \geq 1-\varsigma\right)\right)^{\operatorname{Card}\left(\Im_{N_{k}}\right)} \\
& \geq \exp \left\{-\left(\varepsilon_{N_{k}}+\beta_{N_{k}}\right)(1-\varsigma / 2)\right\} \frac{N_{k}-a_{N_{k}}}{2 a_{N_{k}}} \log ^{d-1} N_{k} \\
& \geq\left(\frac{N_{k}}{2 a_{N_{k}}} \log ^{d} N_{k}\right)^{-(1-\varsigma / 2)} \frac{N_{k}-a_{N_{k}}}{a_{N_{k}}} \log ^{d-1} N_{k} \\
& \geq \frac{a_{N_{k}}}{N_{k}} \cdot \frac{N_{k}-a_{N_{k}}}{2 a_{N_{k}}} \log ^{d-1-d(1-\varsigma / 2)} N_{k} \\
& =\frac{1}{2}\left(1-\frac{a_{N_{k}}}{N_{k}}\right) \log ^{-1+\varsigma d / 2} N_{k} \\
& >\frac{1}{2}(1-\lambda) \log ^{-1+\varsigma d / 2} N_{k} .
\end{aligned}
$$

We also note that

$$
N_{k}=N_{1} \prod_{j=2}^{k} \frac{N_{j}}{N_{j-1}} \leq N_{1} \prod_{j=2}^{k} \frac{1}{\varepsilon\left(1-a_{N_{j}} / N_{j}\right)} \leq N_{1} \varepsilon^{-k+1}(1-\lambda)^{-k+1} .
$$


Thus $\log N_{k} \leq(k-1)|\log (\varepsilon(1-\lambda))|+\log N_{1}$ and the series

$$
\sum_{k=1}^{\infty} \mathrm{P}\left(\max _{J \in \Im_{N_{k}}} S_{J} \delta_{N_{k}} \geq 1-\varsigma\right)
$$

diverges. The Borel-Cantelli lemma implies (20).

Now we consider the case of $p>0$. Let $0<\varsigma<p$. Put $N_{k}=\left[\theta^{k}\right], p>\varepsilon>0$, $\theta=3\left(p^{1 /(d-1)}+1\right)^{d-1}$, and let $m \in \mathbf{N}$. We construct the set of disjoint $d$-dimensional rectangles belonging to the domain $A\left(\theta^{k}\right) \backslash A\left(\theta^{k-1}\right)$ as follows:

$$
\begin{array}{r}
\Im_{\theta^{k}}=\left\{J=(j, i] \in \mathbf{R}_{0}^{d}: i=\left(\varrho^{s_{1}}, \ldots, \varrho^{s_{d-1}}, \frac{\theta^{k}}{\varrho^{\sum_{l=1}^{d-1} s_{l}}}\right), s_{l} \in\left\{1, \ldots,\left[\frac{k \log \theta}{\log \varrho}\right]\right\},\right. \\
\left.s \in \mathbf{N}_{0}^{d-1}, l=1, \ldots, d-1, j=\left(\varrho^{s_{1}-1}, \ldots, \varrho^{s_{d-1}-1}, \frac{\theta^{k-1}}{\varrho^{\sum_{l=1}^{d-1} s_{l}-d+1}}\right)\right\},
\end{array}
$$

where $\varrho=1+p^{1 /(d-1)}$ and $|i|=\theta^{k},|j|=\theta^{k-1}$. The inequality $|i-j|>a_{N_{k-1}}$ holds if $(\varrho-1)^{d-1}\left(\frac{\theta}{\varrho^{d-1}}-1\right)>p, \quad \frac{\theta}{\left(p^{1 /(d-1)}+1\right)^{d-1}}-1>1, \quad$ and $\quad \theta>2\left(p^{1 /(d-1)}+1\right)^{d-1}$.

The latter condition is obvious.

Note that

$$
\operatorname{Card}\left(\Im_{\theta^{k}}\right) \geq \frac{k^{d-1} \log ^{d-1} \theta}{2 \log ^{d-1} \varrho}
$$

Moreover $n=\varepsilon_{N_{k-1}}+\beta_{N_{k-1}}$ for $(j, i] \in \Im_{\theta^{k}}$ and

$$
\begin{gathered}
W_{n}=\sqrt{\frac{\varepsilon_{N_{k-1}}+\beta_{N_{k-1}}}{a_{N_{k-1}}}} S_{(j, i]}, \quad m_{n}(t)=\mathrm{E}\left(\exp \left(t \sqrt{\frac{\varepsilon_{N_{k-1}}+\beta_{N_{k-1}}}{a_{N_{k-1}}}} S_{(j, i]}\right)\right), \\
\lim _{N \rightarrow \infty} \frac{a_{N_{k-1}}}{\varepsilon_{N_{k-1}}+\beta_{N_{k-1}}} \cdot \log \phi\left(\sqrt{\left.\frac{\varepsilon_{N_{k-1}}+\beta_{N_{k-1}}}{a_{N_{k-1}}} \cdot t\right)=\frac{t^{2}}{2}}\right. \\
c_{2}(t)=\frac{t^{2}}{2}=\liminf \psi_{n}(t) / n .
\end{gathered}
$$

Using the inequality $a_{N_{k-1}} / N_{k-1}>p-\varsigma$ we prove, similarly to (21), that

$$
\mathrm{P}\left(\max _{J \in \Im_{\Theta^{k}}} S_{J} \delta_{N_{k}} \geq 1-\varsigma\right) \geq(p-\varsigma)((k-1) \log \theta)^{-d(1-\varsigma / 2)^{2}} \frac{k^{d-1} \log ^{d-1} \theta}{2 \log ^{d-1} \varrho}
$$

for sufficiently large $N_{k-1}$. Thus the series $\sum_{k=2}^{\infty} \mathrm{P}\left(\max _{J \in \Im_{N_{k}}} S_{J} \delta_{N_{k}} \geq 1-\varsigma\right)$ diverges, since its general term is greater than or equal to $(k-1)^{-1+d\left(1-(1-\varsigma / 2)^{2}\right)}$.

An application of the Borel-Cantelli lemma completes the proof of Step 2.

Step 3. Suppose (13) holds. Since

$$
\liminf S_{N} \delta_{N} \geq \liminf S_{N}^{\star} \delta_{N} \geq \liminf D\left(N, a_{N}\right) \delta_{N} \geq \liminf D^{\star}\left(N, a_{N}\right) \delta_{N} \text { a.s., }
$$

it is sufficient to prove that $\liminf D^{\star}\left(N, a_{N}\right) \delta_{N} \geq 1$ almost surely. 
We construct a partition of the $d$-dimensional cube $\left(0,\left[N^{1 / d}\right] \cdot e\right]$ as follows:

$$
\begin{aligned}
\Im_{N}=\{J= & (i, j] \in \mathbf{N}_{0}^{d}: i=\left(i_{1}, \ldots, i_{d}\right), j=\left(j_{1}, \ldots, j_{d}\right), \\
i_{1} \in & \left\{0, a_{N}, \ldots,\left(\left[\frac{N^{1 / d}}{a_{N}}\right]-1\right) \cdot a_{N}\right\}, j_{1}=i_{1}+a_{N}, \\
& \left.i_{k} \in\left\{0,1, \ldots,\left[N^{1 / d}\right]-1\right\}, j_{k}=i_{k}+1 k=2, \ldots, d\right\} .
\end{aligned}
$$

The elements of this partition satisfy

$$
J \in \Im_{N} \Longrightarrow|J|=a_{N}, \quad \operatorname{Card}\left(\Im_{N}\right)=\exp \varepsilon_{N}(1-\zeta) .
$$

Choose an arbitrary $\zeta>0$. Since the random variables $X_{n}$ are independent and the set $\Im_{N}$ consists of disjoint rectangles, we get

$$
\begin{aligned}
\mathrm{P}\left(\max _{J \in \Im_{N}} S_{J} \delta_{N} \leq 1-\zeta\right) & =\left(\mathrm{P}\left(S_{J_{1}} \delta_{N} \leq 1-\zeta\right)\right)^{\operatorname{Card}\left(\Im_{N}\right)} \\
& =O\left(\left(1-\mathrm{P}\left(S_{J_{1}} \delta_{N}>1-\zeta\right)\right)^{N / a_{N}}\right),
\end{aligned}
$$

where $J_{1}=\left(0,\left(a_{N}, 1, \ldots, 1\right)\right] \in \Im$. It follows from Theorem 5.1 that

$$
\lim _{N \rightarrow \infty} \mathrm{P}\left(S_{J_{1}} \delta_{N}>1-\zeta\right)^{1 /\left(\varepsilon_{N}+\beta_{N}\right)}=\exp \left\{-(1-\zeta)^{2}\right\} .
$$

To continue the estimation of (23) we use the inequality $1-x \leq \exp (-x)$ :

$$
\begin{aligned}
& O\left(\exp \left\{-\exp \left(-\left(\varepsilon_{N}+\beta_{N}\right) \cdot(1-\zeta)^{2}+(1-\zeta) \varepsilon_{N}\right)\right\}\right) \\
& \quad=O\left(\exp \left\{-\exp \left\{\beta_{N}\left(\frac{\varepsilon_{N}}{\beta_{N}} \cdot\left(1-\zeta-(1-\zeta)^{2}\right)-(1-\zeta)^{2}\right)\right\}\right\}\right)=O\left(N^{-\sigma}\right)
\end{aligned}
$$

for some $\sigma>1$.

The series $\sum_{N=1}^{\infty} \mathrm{P}\left(\max _{J \in \Im_{N}} S_{J} \delta_{N} \leq 1-\zeta\right)$ converges by (13). The Borel-Cantelli lemma completes the proof.

Remark 5.2. We consider a wider class of increments in Theorems 3.2 and 3.3 as compared to Theorems 2.3 and 2.2 Nevertheless the limits coincide and the normalizing sequences are equivalent for both sets of the results. Therefore the proof of Theorems 3.2 and 3.3 reduces to the proof of the inequality for the upper limit.

Proof of Theorem 3.2. Since $\lim \sup _{N \rightarrow \infty} S_{N}^{\star} \delta_{N} \leq \lim \sup _{N \rightarrow \infty} S_{N} \delta_{N}$, we only need to prove that

$$
\lim \sup S_{N} \delta_{N} \leq \alpha \text { a.s. }
$$

Let $\alpha^{\prime}>\alpha$; then

$$
\mathrm{P}\left(S_{N} \delta_{N}>\alpha^{\prime}\right) \leq \operatorname{Card}\left(A_{N}\right) \cdot \mathrm{P}\left(S_{J} \delta_{N}>\alpha^{\prime}\right),
$$

where $J \subset(0, n] \subset \mathbf{N}_{+}^{d}:|n| \leq N,|J|=a_{N}$. It follows from Theorem 5.1that

$$
\begin{gathered}
\lim _{N \rightarrow \infty}\left(\mathrm{P}\left(S_{J} \delta_{N}>\alpha^{\prime}\right)\right)^{1 / a_{N}}=\inf _{t \in\left(0, t_{0}\right)} \frac{\phi(t)}{\exp \left(t \alpha^{\prime}\right)}=\exp \left(-\frac{1}{C^{\prime}}\right), \\
\lim _{N \rightarrow \infty} \delta_{N}^{-1} a_{N}^{-1}=1,
\end{gathered}
$$

where $C^{\prime}$ is a unique positive solution of equation (9) with $\alpha^{\prime}$ instead of $\alpha$. Let

$$
C^{\prime \prime} \in\left(C^{\prime}, C\right)
$$


then

$\mathrm{P}\left(S_{N} \delta_{N}>\alpha^{\prime}\right) \leq \operatorname{Card}\left(A_{N}\right) \cdot \exp \left(-\frac{C}{C^{\prime \prime}} \log N\right)=\operatorname{Card}\left(A_{N}\right) \cdot \exp (-(1+\mu) \log N)$,

where $\mu=C / C^{\prime \prime}-1>0$. Further we use Lemma 4.3 and continue

$$
\begin{aligned}
& =O\left(\log ^{d-1} N \cdot a_{N} \log ^{d-1} a_{N} \cdot N^{-\mu}\right) \\
& =O\left(\log ^{d} N \cdot \log ^{d-1}(C \log N) \cdot N^{-\mu}\right) .
\end{aligned}
$$

Let $\vartheta>1$ and $N=\left[\vartheta^{r}\right]$. The series $\sum_{r=1}^{\infty} \mathrm{P}\left(S_{\left[\vartheta^{r}\right]} \delta_{\left[\vartheta^{r}\right]}>\alpha\right)$ converges, whence the BorelCantelli lemma implies that $\lim \sup S_{\left[\vartheta^{r}\right]} \delta_{\left[\vartheta^{r}\right]} \leq \alpha$ almost surely. The rest of the proof is the same as that of Theorem 3.1

Proof of Theorem 3.3. It is sufficient to show that $\lim \sup S_{N} \delta_{N} \leq 1$ almost surely. Take an arbitrary $\varepsilon>0$ and estimate the probability

$$
\mathrm{P}\left(S_{N}>(1+\varepsilon) \delta_{N}^{-1}\right)=\mathrm{P}\left(\sqrt{\frac{\log N}{a_{N}}} S_{N}>(1+\varepsilon) \delta_{N}^{-1} \sqrt{\frac{\log N}{a_{N}}}\right) .
$$

Expanding the function $\log \phi$ into the Taylor series for sufficiently large $\tau_{1}$ we prove that

$$
\lim _{N \rightarrow \infty} \frac{a_{N}}{\log N} \log \phi\left(\sqrt{\frac{\log N}{a_{N}}} \cdot t\right)=\frac{\gamma^{2} t^{2}}{2}
$$

in view of relations $\log N / a_{N} \rightarrow \infty$ and (16).

Note that

$$
\delta_{N}^{-1} \cdot \sqrt{\frac{\log N}{a_{N}}} \sim \gamma \sqrt{2} \cdot \log N .
$$

It follows from Theorem 5.1 that

$$
\lim _{N \rightarrow \infty}\left(\mathrm{P}\left(S_{J} \delta_{N}>\alpha_{N}\right)\right)^{1 / \log N}=\inf _{t} \exp \left(\frac{\gamma^{2} t^{2}}{2}-(1+\varepsilon) \gamma \sqrt{2}\right)=\exp \left(-(1+\varepsilon)^{2}\right) .
$$

Thus

$$
\mathrm{P}\left(S_{N}>(1+\varepsilon) \delta_{N}^{-1}\right)=O\left(\log _{+}^{d-1} N \cdot a_{N} \log _{+}^{d-1} a_{N} \cdot N^{1-(1+\varepsilon / 2)^{2}}\right) .
$$

Let $\vartheta>1$ and $N=\left[\vartheta^{r}\right]$. Then the series $\sum_{r=1}^{\infty} \mathrm{P}\left(S_{\left[\vartheta^{r}\right]} \delta_{\left[\vartheta^{r}\right]}>1\right)$ converges. Now the Borel-Cantelli lemma implies that $\lim \sup S_{\left[\vartheta^{r}\right]} \delta_{\left[\vartheta^{r}\right]} \leq 1$ a.s.

The rest of the proof is the same as that of Theorem 3.1.

\section{BIBLIOGRAPHY}

1. J. Steinebach, On the increments of partial sum processes with multidimensional indices, Z. Wahrcsheinlichkeitstheorie verw. Gebiete 63 (1983), 59-70. MR 84i:60043

2. D. Plachky and J. Steinebach, A theorem about probabilities of large deviation with application to queuing theory, Period. Math. Hungar. 6 (1975), 343-345. MR 53:14613

3. M. Csörgő and P. Révész, Strong Approximation in Probability and Statistics, Akadémiai Kiadó, Budapest, 1981. MR 84d:60050

4. K. Prachar, Primzahlverteilung, Springer-Verlag, Berlin-Göttingen-Heidelberg, 1957. MR 19:393b

5. O. I. Klesov, The Háek-Rényi inequality for random fields and the strong law of large numbers, Teor. Veroyatnost. i Mat. Statist. 22 (1980), 58-66; English transl. in Theory Probab. Math. Statist. 22 (1981), 63-72. MR 82i:60050

6. E. K. Titchmarsh, The Theory of Riemann Zeta-Function, 2nd ed., Oxford University Press, Oxford, 1951. MR 13:741c 
7. I. P. Natanson, Theory of Functions of a Real Variable, "Lan", St. Petersburg, 1999; English transl. of the 2nd ed., Ungar, New York, 1961. MR 26:6309

8. M. I. D'yachenko and P. L. Ul'yanov, Measure and Integral, "Faktorial Press", Moscow, 2002. (Russian)

Chair of Mathematics, Department of Physics and Mechanics, St. Petersburg State Technical University, Politekhnitcheskaya Street 29, St. Petersburg 195251, Russia

E-mail address: helgagold_@pochtamt.ru, helga_scher@mailru.com

Received 4/APR/2002

Translated by THE AUTHOR 